Cahiers $d u$ MONDE RUSSE

\section{Cahiers du monde russe}

Russie - Empire russe - Union soviétique et États indépendants

$46 / 4 \mid 2005$

L'invention d'une politique humanitaire

\title{
Willard Sunderland, Taming the Wild Field
}

\section{Juliette Cadiot}

\section{OpenEdition \\ Journals}

Édition électronique

URL : https://journals.openedition.org/monderusse/2895

DOI : 10.4000/monderusse.2895

ISSN : $1777-5388$

Éditeur

Éditions de l'EHESS

Édition imprimée

Date de publication : 1 décembre 2005

Pagination : $945-948$

ISBN : 2-7132-2057-2

ISSN : $1252-6576$

\section{Référence électronique}

Juliette Cadiot, «Willard Sunderland, Taming the Wild Field », Cahiers du monde russe [En ligne], 46/4 2005, mis en ligne le 29 juin 2009, consulté le 03 septembre 2022. URL : http://

journals.openedition.org/monderusse/2895; DOI : https://doi.org/10.4000/monderusse.2895

Ce document a été généré automatiquement le 3 septembre 2022

Tous droits réservés 


\title{
Willard Sunderland, Taming the Wild Field
}

\author{
Juliette Cadiot
}

\section{RÉFÉRENCE}

Willard SUNDERLAND, Taming the Wild Field. Colonization and Empire on the

Russian Steppe. Ithaca, Londres: Cornell University Press, 2004, 264 p.

1 Willard Sunderland présente dans son ouvrage l'histoire d'une région, la steppe, sur presque un millénaire $\left(\mathrm{x}^{\mathrm{e}}-\mathrm{XIX}{ }^{\mathrm{e}} \mathrm{s}\right.$.). Si les quatre cinquièmes du livre concernent de fait la seconde moitié du XVIII et le xIX siècle, le choix du long terme permet de prendre la mesure des changements et des différentes phases d'accélération dans la transformation du territoire. Ce dernier s'étend au nord et au sud à partir du Danube, entre la Caspienne et la mer Noire, jusqu'à l'Oural, allant de Kichinev et Odessa jusqu'à Vladikavkaz au sud et Orenbourg au nord. La steppe est aujourd'hui divisée entre l'Ukraine, la Moldavie, la Fédération russe et le Kazakhstan. Le travail de Sunderland se situe au croisement de plusieurs renouvellements historiographiques concernant aussi bien l'histoire générale et régionale de l'empire de Russie que l'histoire des confins, des zones de frontières (borderlands).

2 L'auteur se propose de confronter l'histoire de cette zone géographique, originairement consacrée au pastoralisme et au nomadisme, et qui, à la fin du XIX ${ }^{\mathrm{e}}$ siècle, est dédiée à l'agriculture sédentaire, à d'autres expériences de colonisation, soit américaines, soit européennes. L'un des grands apports du livre consiste en la volonté d'éclairer l'épopée impériale russe à l'aune d'autres historiographies. Ainsi Sunderland introduit-il la notion " d'impérialisme » pour parler de l'ensemble de la période, définissant ce dernier comme la formation d'un empire et celui-ci comme la création d'une société subordonnée. L'utilisation de ce terme paraît néanmoins superflue, même s'il renvoie à une nouvelle tendance historiographique qui cherche à souligner le caractère nécessairement violent et destructeur, pour les sociétés autochtones, de l'intrusion d'un nouveau pouvoir 
étatique et de populations nouvelles. Dans le corps de l'ouvrage, Sunderland est plus nuancé, montrant combien les discours sur la colonisation, l'empire et l'impérialisme (qui n'émerge en tant que terme que dans les années 1870) ont évolué et combien les acteurs de la colonisation russe ont tenté de décrire leurs expériences en fonction de ce qui se passait ailleurs, autant pour s'y identifier que pour s'en démarquer. L'historien note bien le changement introduit dans la seconde moitié du XIX ${ }^{e}$ siècle avec la multiplication des conquêtes coloniales et l'émergence d'une idéologie particulière, mais celui-ci s'applique moins à la steppe, administrativement et économiquement bien intégrée au centre, qu'à l'Asie centrale notamment. L'auteur analyse largement les changements de vocabulaire (migration, peuplement, colonisation...). Il montre comment, à partir de la seconde moitié du XIX siècle - époque d'uniformisation administrative accélérée par les Grandes Réformes -, les régions de la steppe furent redéfinies dans la mythologie nationale comme un entre-deux, à la fois territoire intérieur et frontière orientale.

3 Le livre a donc pour ambition de décrire le phénomène d'intégration des régions de la steppe à l'empire de Russie. Du fait du choix des sources - chroniques, récits de voyage, études ethnographiques et historiques, archives centrales et provinciales -, le parti pris de l'auteur est de se placer du point de vue du colonisateur. En réalité, les colonisés sont peu présents dans cette histoire, si ce n'est par la mention des révoltes, départs en émigration et, assez vite, la mise en place d'institutions autonomes. Par contre, Sunderland s'applique à construire une histoire, tant institutionnelle que des représentations, du pouvoir russe sur la steppe. Aussi son étude se présente-t-elle également comme une histoire générale de l'empire - notamment en ce qui concerne la création d'une idéologie nationale impériale-, qui place le fait colonial au centre de la définition d'une particularité nationale russe.

4 Le premier chapitre retrace rapidement l'histoire du territoire de la steppe, depuis sa mention comme champ (field) dans les chroniques anciennes de la Rus', à l'arrivée des Mongols et sa nouvelle appellation de « champ sauvage » (« the wild field» du titre) après la reconquête de Kazan dans la seconde moitié du Xvi ${ }^{\mathrm{e}}$ siècle. Les différentes tentatives de pacification de ce territoire afin d'en faire une ligne défensive qui ne cesse d'avancer aboutissent à l'émergence du terme de "steppe » à la fin du XVII e siècle. Le territoire du sud de la Moscovie est alors conceptualisé comme un espace vide à développer. Si les princes de Moscovie avaient recherché la sécurité, les profits du commerce et entrepris quelques opérations de conversion forcée, Pierre le Grand et ses successeurs, créateurs de l'empire, ont voulu davantage. Selon Sunderland, un nouvel impérialisme émerge avec Pierre, fondé sur la rationalité, la transformation des territoires et des peuples, et qui se manifeste par l'organisation des grandes expéditions scientifiques. Au milieu du $\mathrm{XVIII} \mathrm{e}^{\mathrm{e}}$ siècle, la steppe est donc devenue un lieu à peupler, le mercantilisme propre à la période impliquant d'organiser son développement économique.

Le second chapitre, intitulé " La colonisation par les Lumières ", s'intéresse à la seconde moitié du XVIII siècle. Il décrit en particulier la politique de conquête de Catherine II au cours de guerres successives avec l'empire ottoman, ainsi que sa volonté de peupler la steppe non seulement de Russes et d'Ukrainiens issus des provinces centrales, cosaques ou paysans d'État, mais de colons (kolonisty) étrangers: Allemands, Bulgares, Grecs... Sunderland décrit, comme dans chacun des chapitres suivants, l'évolution institutionnelle, l'arrivée des différents migrants, la collecte de renseignements, les épisodes de conquête (plus les départs consécutifs de nombreuses populations 
autochtones) et de révoltes (celle des Bachkirs, la rébellion de Pugačev chez les cosaques). Enfin il utilise, trop rapidement à notre goût, les récits des migrants euxmêmes ou des administrateurs locaux, qui font apparaître la réalité sur le terrain de la rencontre des Russes, fuyards ou mal organisés, avec leurs nouveaux territoires et leurs habitants. Il était essentiel de resituer cette réalité chaotique afin de mieux apprécier l'ampleur des efforts qui furent nécessaires à une colonisation agricole qui s'étendit sur deux siècles.

6 «Une colonisation bureaucratique » (troisième chapitre) étudie le premier quart du XIX ${ }^{e}$ siècle, période d'intense colonisation (entre 1796 et 1835, on compte 1,7 million de nouveaux migrants). Le romantisme et l'idée de rationalisation de l'État s'entremêlaient pour faire de la steppe une colonie importante que l'on souhaitait contrôlée. Dans le chapitre IV, Sunderland décrit la période suivante, avec la formation en 1837 d'un ministère des Domaines qui devient de fait le ministère de la colonisation. L'auteur montre la poursuite d'une colonisation ethniquement diversifiée et que les pouvoirs publics cherchent toujours à mieux encadrer, puis le changement introduit par l'émancipation du servage. Plutôt que de continuer à coloniser, il s'agit désormais d'améliorer les conditions agricoles et économiques des paysans de la Russie centrale comme de la périphérie. En outre, dans le cadre de l'unification administrative liée aux Grandes Réformes, les régions de la steppe et leurs populations (colons, Bachkirs et en partie les cosaques) perdent une part de leur autonomie et se trouvent intégrées aux provinces intérieures.

7 Le dernier chapitre, qui porte sur la fin $\mathrm{du}$ xIx siècle, clôt cette histoire de l'incorporation de la steppe par la création d'institutions spécialisées plus compétentes dans la colonisation, mais aussi par la substitution de la Sibérie à la steppe comme grande zone de peuplement. Sunderland souligne enfin les ambiguïtés de la construction nationale russe. Si les études ethnographiques et statistiques n'ont cessé de se multiplier, l'ambiguïté demeure néanmoins chez les savants: la Russie a-t-elle entrepris une colonisation intérieure ou bien a-t-elle été, à l'instar des grandes puissances européennes, une puissance impérialiste?

- Le livre de Sunderland, par son ambition, l'ampleur des sources utilisées, sa richesse d'analyse et son inscription dans les débats historiographiques les plus contemporains, constitue l'un des exemples les plus achevés de la réécriture en cours de l'histoire de la Russie, qui prend pleinement en compte sa dimension impériale. La volonté de rompre avec la vision d'une colonisation organique, d'une incorporation "naturelle » des terres qui constituèrent l'empire russe puis l'URSS, permet de replacer au centre du récit l'extériorité originelle des territoires et des populations conquises. Mais, en se fondant sur les seules archives russes et en restituant le seul point de vue du colonisateur, en particulier ses représentations de la steppe, Sunderland parle finalement surtout de la construction nationale russe. Il ouvre cependant la voie pour réélaborer aujourd'hui une méthodologie qui permettrait de décrire dans le détail comment un pouvoir impérial s'est imposé et comment les populations locales, si diverses soient-elles, ont réagi. 